\title{
Gestión de la seguridad alimentaria frente al covid-19, en el Perú, 2020
}

\author{
Jacinto Villanueva Pante \\ jacipante2018@gmail.com \\ Universidad Cesar Vallejo \\ Lima-Perú
}

\section{RESUMEN}

Debido a la debilidad de la capacidad de gestión en la seguridad alimentaria enfocado en la población en los niveles de gobierno, en los niveles de gobierno, gestión regional provinciales y distritales que no han prevenido la seguridad alimentaria en las madres de familia de niños menores de 5 años, que se han enfocado en las unidades ejecutoras regionales en la que hay mucha deficiencia no han efectuados los presupuestos asignados a las familias de menores recursos más bien en la demanda en la prevención de la sanidad sanitaria a pesar del agotamiento y saturación hospitalaria, a la falta de camas unidad de cuidados intensivos en las regiones debido a la pandemia del Covid 19 y las olas de la enfermedad debida a las mutaciones Beta, Gamma y Delta en diversas regiones se ha puesto diversas realidades que se va dar en la problemática de la situación de la seguridad alimentaria en la desnutrición social de las madres de familia con los niños menores de 5 años ante un eminente cambio climático, en el Perú es altamente vulnerable a los riesgos ocasionados por los fenómenos naturales y efectos del cambio climático, como la inundaciones, heladas, huaycos, sequías, friajes, entre otros. Los eventos perdida de trabajo, cambio climático, capacidad de gestión de los gobiernos regionales son catastrófico que afecta la producción de alimentos y que empobrece a las familias que provisiona en la falta de alimentos, en la alza de los alimentos y también a la calidad proteica de los mismos, en la familia desprotegida de menores recursos madres con niños menores de 5 años se ven en situación de vulnerabilidad. Se traducido por la mala gestión gubernamental de las regiones se da también en una inadecuada gestión en la alimentación.

Palabras Claves: gestión gubernamental; covid-19; seguridad alimentaria; unidad de cuidados intensivos 


\title{
Food security management in the face of covid-19, in Peru- 2020
}

\begin{abstract}
Due to the weakness of management capacity in food security focused on the population at the levels of government, at the levels of government, provincial and district regional management that have not prevented food security in mothers of children under 5 years, which have focused on regional implementing units in which there is much deficiency have not made the budgets allocated to low-income families rather on demand in the prevention of sanitation despite the exhaustion and hospital saturation, The lack of intensive care unit beds in the regions due to the Covid 19 pandemic and the waves of the disease due to Beta, Gamma and Delta mutations in various regions has put various realities that will be given in the problematic situation of food security in the social malnutrition of mothers with children under 5 years of age in the face of an eminent climate change, Peru is highly vulnerable to the risks caused by natural phenomena and the effects of climate change, such as floods, frosts, landslides, droughts, cold spells, among others. The events lost work, climate change, management capacity of regional governments are catastrophic that affects food production and impoverishes families that provides in the lack of food, in the rise of food and also the protein quality of the same, in the unprotected family of lower resources mothers with children under 5 years are in a vulnerable situation. This is translated by the poor governmental management of the regions, which also results in an inadequate food management.
\end{abstract}

Keywords: government management; covid-19; food security; intensive care unit.

Artículo recibido: 10 Setiembre. 2021 Aceptado para publicación: 15 Octubre. 2021 Correspondencia: jacipante2018@gmail.com Conflictos de Interés: Ninguna que declarar 


\section{INTRODUCCIÓN}

Roma en 2002 en el Foro de Soberanía Alimentaria celebrado, "la soberanía alimentaria es el derecho de los pueblos, comunidades y países a definir sus propias políticas agrícolas, pesqueras, alimentarias y de tierra que sean ecológica, social, económica y culturalmente apropiadas a sus circunstancias únicas. Esto incluye el verdadero derecho a la alimentación y a producir los alimentos, lo que significa que todos los pueblos tienen el derecho a una alimentación sana, nutritiva y culturalmente apropiada, y a la capacidad para mantenerse a sí mismos y a sus sociedades"

Involucra los siguientes "los factores de la producción agroalimentaria; la agrobiodiversidad y semillas; la investigación y diálogo de saberes; la producción, transformación, conservación, almacenamiento, intercambio, comercialización y consumo; así como la sanidad, calidad, inocuidad y nutrición; la participación social; el ordenamiento territorial; la frontera agrícola; los recursos hídricos; el desarrollo rural y agroalimentario; la agroindustria, empleo rural y agrícola; las formas asociativas y comunitarias de los microempresarios, microempresa o micro, pequeños y medianos productores, las formas de financiamiento; $y$, aquéllas que defina el régimen de soberanía alimentaria"

Guzmán, J. (2017). La inadecuada gestión de los recursos naturales, el desempleo rural, la desigualdad, los conflictos civiles, etc.), políticas (forma en el que administran el poder, tanto dentro como fuera de los Departamentos, etc.), económica (baja capacidad para generar ingresos, etc.) y ambientales (mala utilización de los suelos, a la inadecuada distribución de las tierras disponibles, etc.)”

Leclercq-Barriga M, Liévano-Fiesco, García-Londoño, Lombo, Castro, Morales (2020) Concluyó que las instituciones aceptan los recursos con la finalidad de entregarlos sin tener la finalidad de brindar u orientar sobre el balance alimentario para las familias, ya que para ellas lo más importante es recibir los recursos. Además, hasta la fecha del estudio no se habían realizado algún proceso de formación y de gestión con el fin de mejorar la adecuación nutricional y hábitos alimenticios saludables (45).

De acuerdo al Plan Nacional de Gestión de Riesgo y Adaptación al Cambio Climático del Sector Agrario, en Perú la helada es la ocurrencia de una temperatura igual o menor a $0^{\circ}$ C, a un nivel de 1,5 a 2 m sobre el suelo. Por su parte, el friaje está definido como la incursión de masas de aire polar asociada a una repentina disminución de la temperatura 
del aire en la selva peruana, pudiendo disminuir hasta $15^{\circ} \mathrm{C}$ en solo horas (Marengo, 1984). Afecta a la agricultura en general

A nivel nacional, de acuerdo al Plan de Gestión de Riesgo y Adaptación al Cambio Climático en el Sector Agrario 2012-2021, al 2030 se esperaría, que en la Costa norte y central las temperaturas se incrementen hasta en $2{ }^{\circ} \mathrm{C}$ y $1{ }^{\circ} \mathrm{C}$, respectivamente, las precipitaciones aumenten entre $10 \%$ y $20 \%$ y la disponibilidad de agua sería menor afectando productos como el maíz amarillo duro y la papa. Por su parte, en la Sierra se proyecta ligeros aumentos en la temperatura, hasta $1,5^{\circ} \mathrm{C}$ y en algunas zonas, se presentaría disminuciones considerables de lluvias, mayores al 28\%, lo que generaría déficits de rendimiento que alcanzaría entre el 12\% y 15\%, lo que llevaría a que los ciclos de vida se acorten hasta en 13 días y las cosechas se adelanten. En la Sierra, el escenario es distinto, se proyectan cambios ligeros en el clima.

Los objetivos priorizados en la Resolución Ministerial N0265-2012-AG, 06. Octubre del 2012. Son utilizar y asegurar el consumo adecuado de alimentos inocuos y nutritivos, respetando los hábitos alimenticios y la interculturalidad de cada región. y Asegurar servicios de agua segura y saneamiento integral: educación sanitaria, infraestructura y gestión. Y el objetivo: Estabilidad Garantizar medidas de adaptación a manifestaciones del cambio climático y prevención y contingencias frente a eventos como plagas y enfermedades, factores de mercado, situaciones de conflicto y otros que pudieran generar crisis de inseguridad alimentaria. Fortalecer las capacidades institucionales de planeamiento, prevención y manejo de situaciones de crisis de inseguridad alimentaria a nivel nacional, regional y local. Esta estrategia está orientada a promover la cultura de la gestión de riesgos ante situaciones de crisis de seguridad alimentaria y fortalecer el desarrollo del conocimiento y las capacidades de los gestores públicos para hacer frente dichas situaciones, así como para desarrollar mecanismos que permitan implementar medidas de prevención y acciones de respuesta rápida y eficaz.

Desarrollar un sistema de vigilancia ante crisis de seguridad alimentaria frente a manifestaciones del cambio climático y eventos como plagas y enfermedades, factores de mercado, situaciones de conflicto y otros que pudieran generar crisis de inseguridad alimentaria. Esta estrategia está orientada a diseñar y promover la implementación de políticas que permitan contar con sistemas de vigilancia y de comunicación, así como a desarrollar e impulsar los instrumentos técnicos y de gestión de los procesos de 
adaptación, prevención, reducción, preparación y respuesta que permitan reducir los impactos negativos en la seguridad alimentaria ante escenarios de crisis.

Gestionar mecanismos financieros para la implementación de la Estrategia Nacional de Seguridad Alimentaria y Nutricional (ENSAN).

Lo que busca esta estrategia es diseñar programas presupuestales vinculados a la Estrategia Nacional de Seguridad Alimentaria y Nutricional y gestionar otros mecanismos financieros que permitan implementar la Estrategia.

Según datos del instituto Nacional de Estadística INEI (2020) en el Perú hay 9 millones 820 mil pobres de esto se sitúa en la zona rural $45.7 \%$ y en la zona urbana 20.0 , que ha aumentado en casi 10 años en 9.9 de 20.1 a 30.1 está referido en el cuadro 1 este índice se vio directamente afectado por la pandemia de Covid-19, hecho que obligó a las madres de familia con niños menores de 5 años a impactar en los hogares en la alimentación de los niños y sus capacidades y adecuar el desarrollo cognitivo.

Figura 1. Situación de pobreza en el Perú causado por el Covid-19

\section{Crece la población en situación de pobreza}

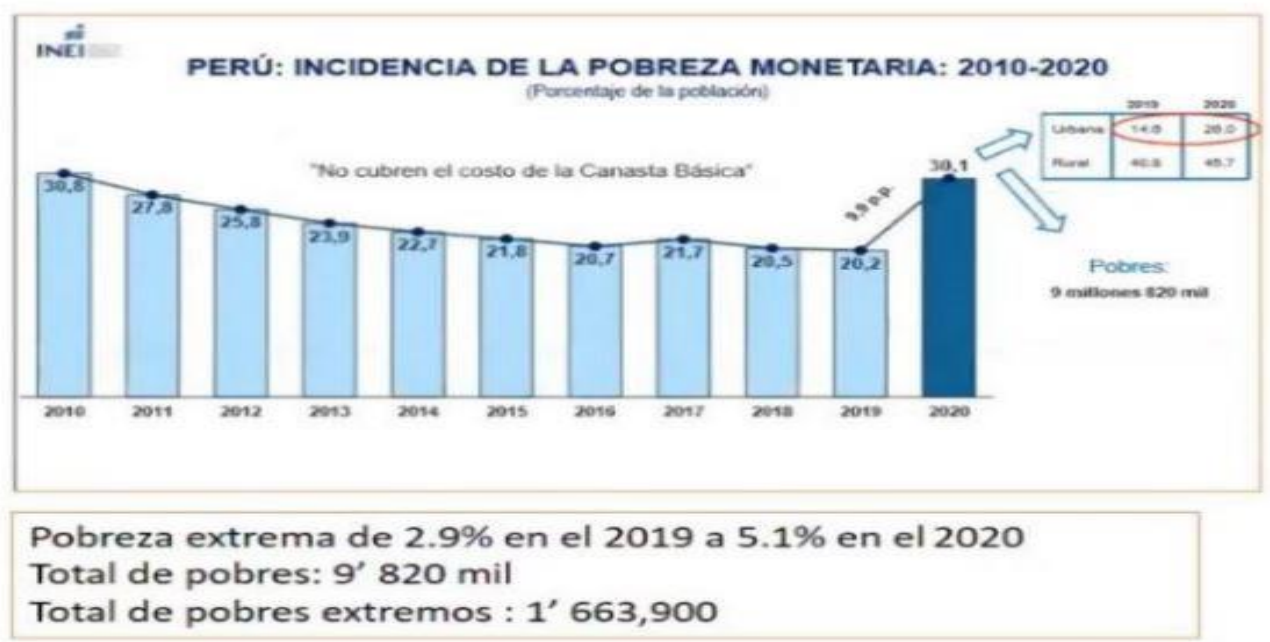

Fuente: INEI (2020).Instituto nacional de Estadística del Perú

\section{ESTRATEGIAS METODOLÓGICAS O MATERIALES Y MÉTODOS}

La investigación es de tipo básica también conocida como investigación fundamental, exacta o investigación pura, que se ocupa del objeto de estudio sin considerar una aplicación inmediata, pero teniendo en cuenta que, a partir de sus resultados y descubrimientos, pueden surgir nuevos productos y avances científicos (Cívicos y Hernández, 2007; Padrón, 2006). 
El diseño es No experimental, Transversales o Transeccionales porque según Hernández Sampieri et al. (2014) son diseños experimentales estudios que se llevan a cabo sin manipular conscientemente variables y donde solo se observan fenómenos en su entorno natural y luego se analizan (p.154) en la investigación no se manipulan las variables solo se extrae la información para su análisis.

Lo que hacemos en la investigación no experimental es observar fenómenos tal como se dan en su contexto natural, para analizarlos (The SAGE Glossary of the Social and Behavioral Sciences, 2009b).

\section{Políticas Públicas para incrementar la capacidad de la población de tener acceso a alimentos de calidad}

La pérdida de los derechos a la alimentación de calidad puede también verse afectada por la incapacidad de ciertos grupos poblacionales de acceder a alimentos que están disponibles en el mercado. Esta situación puede darse por dos factores esenciales en los cuáles el Estado puede intervenir: (1) altos niveles de especulación en los precios de los alimentos; y (2) bajos y variables ingresos en algunos segmentos de la población.

\section{Capacidades de gestión de los gobiernos locales en el Perú durante la pandemia}

\section{Covid 19}

Debido a los informes en medios de la Defensoría del Pueblo (Gutiérrez, 2020) dan cuenta de la poca capacidad de gestión de los Gobiernos Regionales ante la crisis, por ejemplo, el Gobierno Regional de Arequipa a la actualización del portal de transparencia del Ministerio de Economía y Finanzas el 8 de abril del 2020 ha ejecutado $1.6 \%$ de un presupuesto de S/. 6,900.523; el Gobierno Regional de La Libertad ejecutó el 7.9\% de S/. 14,619.156, el titular del sector plantea la necesidad de mejorar las competencias de los Gobiernos Regionales frente a las actividades priorizadas.

La gestión en estas presentan problemas en el desarrollo de planes articulados para hacer frente a la crisis, intereses de por medio, algunas regiones ya muestran algunos problemas en el desarrollo de sus competencias, una de las disposiciones es la distribución de canastas de productos básicos para ayudar a familias en situación de vulnerabilidad, competencia de los municipios provinciales y distritales, la Contraloría General de la República ha informado sobre deficiencias en esta distribución, detectando demoras en la adquisición de los productos, padrones de beneficiarios incompletos y escasa focalización 
de las familias vulnerables, las observaciones de este organismo en un municipio nos da un claro ejemplo de las deficiencias en la gestión de estas acciones son:

- No se ha culminado de sincerar el padrón de los hogares vulnerables a ser beneficiados. - La cantidad de productos incluidos en las canastas básicas familiares es inferior a lo señalado por la Entidad.

- No se viene cumpliendo con registrar proceso de contratación de canastas básicas familiares.

La Contraloría General de la República ha venido emitiendo informes por cada municipio dentro de su campaña "Emergencia Sanitaria 2020" en los que identifica situaciones que son informadas a las autoridades competentes de generar cambios, adecuaciones o iniciar procesos de mejora. Para saber más sobre este organismo visite.

La necesidad de la gestión pública y la fortaleza de sus instituciones para la gestión de todas las necesidades que se van multiplicando se hacen visibles en estos meses, parece una desconexión entre los liderazgos locales y los gestores institucionales con los requerimientos y necesidades a ser afrontados, también en una diferencia en el entendimiento de los números de la pandemia y lo que está detrás de ellos, las necesidades de sectores específicos se hacen más visibles en las exigencias de respuestas y solución a sus problemáticas, ya no por la reivindicación de derechos o libertades, sino por la crisis humanitaria en la que pueden verse envueltos; es cierto que no estábamos preparados para las medidas de aislamiento que la emergencia requiere.

\section{Perspectivas brindadas por el Covid-19 frente a la Inseguridad alimentaria}

\section{Realidad de la gestión del gobierno local en la Pandemia}

Sistema Informático Nacional de Defunciones (Sinadef) del Ministerio de Salud del Perú. Solo entre abril y mayo del 2020, el Sinadef registró un excedente de 17,814 personas fallecidas en comparación con el 2019.Desde una perspectiva del conflicto social, la pandemia ha traído consigo nuevos problemas o acentuado los ya existentes. basadas en la salud y la economía, y los posibles cambios de visión sobre la realidad del país. La comprensión de estos elementos solo es posible si nos preguntamos por el contexto, por aquello que está cambiando sensiblemente a nuestro alrededor y que será decisivo en la forma cómo encaremos los conflictos. Las afectaciones a la salud y la vida la pandemia por el COVID-19 ha puesto en evidencia la debilidad y precariedad del sistema de salud en el país y ha incrementado las desigualdades preexistentes, afectando a la población 
más vulnerable. Al 30 de junio, la cifra de personas contagiadas con el virus es de 285,213 a nivel nacional, según el Ministerio de Salud (Minsa), lo que nos coloca como el sexto país con mayor número de contagios a nivel mundial. Los ámbitos geográficos con mayor número de personas contagiadas son Lima, con 158 mil; seguida del Callao, con 18 mil; Piura, con 17 mil; Lambayeque, con 13 mil; entre otros. El número de personas fallecidas alcanza los 967712, el $46.4 \%$ del total de los decesos se produjeron en Lima. La propagación del COVID-19 ha tenido un mayor impacto en la salud de las personas adultas mayores. De acuerdo con las cifras del Minsa, del total de personas fallecidas por el virus, el $68.9 \%$ pertenece a este sector de la población. Antes de la propagación del coronavirus, las personas adultas mayores eran consideradas un grupo poblacional vulnerable que requería una protección especial para salvaguardar su vida, salud, integridad y dignidad. En el contexto de la pandemia esta vulnerabilidad se ha incrementado y se han generado nuevas situaciones de riesgo que los coloca como la población más afectada en el número de decesos. Con relación al sexo de los contagiados, el $42 \%$ son mujeres y el $58 \%$ son hombres. Sin embargo, esta brecha entre hombres y mujeres incrementa con relación a los fallecidos. El 71\% de los decesos pertenecen al sexo masculino, mientras que el $29 \%$ al sexo femenino. Esto pone en evidencia que existe un sector de la población que corre mayor riesgo de letalidad con el COVID19. Por su parte, las organizaciones de representación indígena han cuestionado las cifras proporcionadas por las direcciones regionales, argumentando que estas no coinciden con las cifras de los centros de salud de las localidades y no se estaría brindando atención a las comunidades más alejadas. Sostienen que las comunidades nativas carecen de implementos de bioseguridad y artículos de higiene, lo que incrementa la probabilidad de contagio.

PNUD. (2020). Vulnerabilidades, más allá de la pobreza. El Perú antes del brote del COVID-19 era un país vulnerable. El 65\% de los hogares registraba por lo menos algún tipo de vulnerabilidad (laboral, alimentaria, monetaria, financiera e hídrica). Estas vulnerabilidades, como es evidente, se han ido acentuando durante la pandemia, agravadas por la mayor pobreza, desempleo, informalidad.

\section{CONCLUSIÓNES}

Las capacidades, debido a la limitada competitividad en los cuadros técnicos profesionales de las regiones para afrontar la gestión del cambio climático; 
La pandemia del COVID-19 ya está afectando directamente los sistemas alimentarios, mediante impactos en la oferta y demanda de alimentos, e indirectamente pero igualmente importante- a través de la disminución del poder adquisitivo y la capacidad de producir y distribuir alimentos, y la intensificación de las tareas de atención, todo lo cual tendrá repercusiones diferenciadas y afectará más fuertemente a los pobres y vulnerables.

La experiencia adquirida hasta ahora con el brote del COVID-19 proviene de países desarrollados e industrializados (China, Corea del Sur, Italia, entre los más afectados), y ya hay una clara afectación negativo del brote en los mercados bursátiles, la producción industrial y la demanda de petróleo. Sin embargo, es difícil predecir el impacto a largo plazo en la economía en su conjunto y en la SAN, especialmente en los países de bajos ingresos, con base en la experiencia actual. Pero las señales de desaceleración económica y la interrupción de las cadenas de valor de los alimentos, son evidentes.

Mejorar la calidad y esperanza de vida, y aumentar las capacidades y potencialidades de la población en el marco de los principios y derechos que establece la Constitución para las madres con niños menores de 5 años.

De igual manera, se cuenta con el Programa de Modernización de Gestión Municipal y el Plan de Incentivos a la Mejora de la Gestión y Modernización, instrumentos que abordan la gestión de residuos y la reducción del riesgo de desastres a nivel municipal y no de la gestión nutricional de la seguridad alimentaria.

Su principal aporte es que contribuye a poner en la agenda de los gobiernos locales la gestión de residuos y del riesgo de desastres, aunque la integración del cambio climático resulta de manera indirecta que puede impactar en la seguridad alimentaria.

\section{REFERENCIAS}

Castillo, Marlene (2020). "Contagio COVID-19 en Lima se atenúa y galopa en Amazonía Indígena del norte". En Forum Solidaridad Perú. Consulta: 25 de junio de 2020. Disponible en: http://www.psf.org.pe/institucional/2020/06/contagio-COVID-19en-lima-se-atenua-y-galopa-en-amazoniaindigena-del-norte-por-marlene-castillo/.

Datos de la Contraloría sobre la Municipalidad de Lambayeque registrados en su informe https://apps8.contraloria.gob.pe/SPIC/srvDownload/ViewPDF?CRES_CODIGO=2 020CSIL43000100\&TIPOARCHIVO=R E, https://www.contraloria.gob.pe/ 
El gobierno elaboró un Plan para afrontar la pandemia del COVID-19 https://cdn.www.gob.pe/uploads/document/file/505245/resolucion-ministerial-0392020-MINSA.PDF

Las regiones de La Libertad y Lambayeque han recibido indicaciones por ejemplo para una mayor gestión de los recursos de emergencia para el COVID-19 https://www.defensoria.gob.pe/wp-content/uploads/2020/04/Reporte-regionaldeLambayeque-y-La-Libertad-09042020.pdf

Leclercq-Barriga M, Liévano-Fiesco, García-Londoño, Lombo, Castro, Morales. Caracterización de la Vulnerabilidad Alimentaria en familias beneficiarias de la Fundación Banco Arquidiocesano de Alimentos. Bogotá, Colombia, 2005 [Internet]. Redalyc.org. 2020[citado 5 noviembre 2020]. Recuperado de: https://www.redalyc.org/articulo.oa?id=49913105

Joaqui Guzmán, N. (2017). Causas que Conllevan a una Inseguridad Alimentaria. Memorias De Congresos UTP, 186-193. [Citado 28 febrero 2021]Recuperado de: https://revistas.utp.ac.pa/index.php/memoutp/article/view/1492

Soberanía Alimentaria. En línea. Consultado el 12 de septiembre de 2012. Disponible en: http://www.soberaniaalimentaria.com/textos/ForoSobAlimentaria2002.htm

Marengo,(1983)http://www.observatoriocambioclimatico.org/system/files/publicacione s/archivos/PLANGRACC-A.pdf

Aprobado por Resolución Ministerial Nº265-2012-AG, 06. Octubre del 2012.

Amanda Lee *, Dori Patay, Lisa-Maree Herron, Ella Parnell Harrison y Meron Lewis. (2021). Asequibilidad de dietas actuales, saludables, más equitativas y sostenibles por área de desventaja socioeconómica y lejanía en Queensland: conocimientos sobre la elección de alimentos. Sotavento et al. Revista internacional para la equidad en salud, 17. Obtenido de https://doi.org/10.1186/s12939-021-01481-8

Amy Molotoks, Pete Smith, Terence Dawson. (2021). Impactos del uso de la tierra, la población y el cambio climático en la seguridad alimentaria mundial. Food and Energy Segurity, 1-20. doi:https://doi.org/10.1002/fes3.266

Ana Beatriz Pinto de Almeida Vasconcellos, Leides Barroso Azevedo de Moura. (2018). Seguridad alimentaria y nutricional: análisis sobre la situación de la descentralización de la política pública nacional. Cadernos de Saúde Pública, 1-13. doi: 10.1590/0102-311X00206816 
Arturo Elias Alexei Egocheaga Díaz, Cindy Flor Córdova Humbo. (2012). Nivel de seguridad alimentaria asociado a pobreza y apoyo alimentario en hogares de un Asentamiento Humano de Puente Piedra, Lima. Lima-Peru: E.A.P nutrición. Obtenido de http://cybertesis.unmsm.edu.pe/bitstream/handle/20.500.12672/11537/Cordova_hc. pdf? sequence $=1 \&$ isAllowed $=y$

Aulestia-Guerrero, E. M. (2020). Una mirada hacia la inseguridad alimentaria sudamericana. Ciência \& Saúde Coletiva, 2507-25017. doi:http://dx.doi.org/10.1590/1413-81232020257.27622018

Baquero, F. S. (2008). Políticas públicas y la nueva situación en los precios internacionales de los alimentos. Oficina Regional FAO para ALC, 1-25. Obtenido de

http://www.fao.org/fileadmin/templates/fmpp/documents/Politicas_Publicas_nueva _situacion_precios_int.pdf

Baquero, F. S. (2009). Politicas públicas para enfrentar la crisis alimentaria. Oficina Regional para la FAO en América Latina y el Caribe, 1-17. Obtenido de http://www.fao.org/fileadmin/templates/fmpp/documents/Capitulo_III_Panorama_ 2009_RLC.pdf

Cullen, M. T. (2019). El estado de la seguridad alimentaria y la nutrición en el mundo 2019. Organización de las Naciones Unidas para la Alimentación y la Agricultura, $1-256$.

Obtenido

de

file:///C:/Users/User/Documents/SEMINARIO\%20V/TRABAJOS_INV5/trabajo6/ politicas/ca5162es.pdf

Edgar Alfredo Nande Vázquez, Omar Alejandro Pérez Cruz, Saúl Martínez González, José Ernesto Rangel Delgado. (2019). Seguridad alimentaria y obesidad: Un análisis comparativo entre China-México-EU. Portes, revista mexicana de estudios sobre la cuenca del Pacífico, 105-137. Obtenido de cpnade@ucol.mx

Elena Mukhina, Tatyana Medvedeva, Vladimir Kudinov, Evelina Farvazova, y Alia Esembekova. (2021). Enfoques conceptuales para la investigación de los sistemas agroalimentarios modernos. Interagromash 2021, 13. Obtenido de https://doi.org/10.1051/e3sconf/202127313005 
Gusztáv Nemesa,*, Yuna ChiffoleauB,*, Simona Zollet C, Martin CollisonD, Zsó fi a Benedeka, Fedele Colantuonomi, Arne Dulsrud F, Mariantonietta Fioremi, Carolin Holtkampgramo, Tae-Yeon Kimh, Monika KorzunI, Rafael Mesa-Manzanoj, Rachel Reckingerk, Irune Ru. (2021). El impacto de COVID-19 en los sistemas alimentarios alternativos y locales y el potencial para la transición de la sostenibilidad: perspectivas de 13 países. Producción y consumo sostenible, 591-599. Obtenido de https://doi.org/10.1016/j.spc.2021.06.022

Manning, L. (2020). Desarrollo de una seguridad alimentaria modelo de riesgo de verificación. British Food Journal, 115(4), 575-589. doi: 10.1108 / 00070701311317856

María del rosario granados sánchez, Javier galán figueroa, Juan antonio leos rodríguez. (2020). Volatilidad en los precios de los cereales básicos y su impacto en la seguridad alimentaria. México, 1995-2018. Revista de Ciencias Sociales y Humanidades, 79105. doi: http://dx.doi.org/10.20983/noesis.2020.2.4

Pablo Alarcón, Paula Domínguez, Eric Fèvre, Jonathan Rushton. (Abril de 2021). La importancia de un enfoque de sistemas alimentarios para los países de ingresos bajos y medios y las economías emergentes: una revisión de las teorías y sus Relevancia para el control de enfermedades y la desnutrición. frontiers in sustainable Foods systems, 1-16. doi:10.3389 / fsufs.2021.642635

Patrick Caron a, Martin van IttersumB, Tessa Avermaete C, Gianluca Brunori D, Jessica Fanzo mi, Ken Giller B, Etienne Hainzelin F, John Ingramgramo, Lise Korsten h, Yves Martin-Pr'ével I, Moisés Osiru j, Cheryl Palmk, Marta Rivera Ferre 1, Mariana Rufin. (2021). Declaración basada en la $4^{\text {a }}$ conferencia internacional sobre seguridad alimentaria mundial - Diciembre de 2020: desafíos para una agenda de investigación disruptiva. Seguridad alimentaria mundial 30, 2. Obtenido de https://doi.org/10.1016/j.gfs.2021.100554

Tatiana Lena Aguilar Torrico, Silvina Papagno, Roberto Mariano, Javier Vitale, Paola Aceituno O. (2018). Hacia una prospectiva latinoamericana un abordaje desde lo agrícola y lo alimentario. revista de estudios políticos y estratégicos, 184-192. Obtenido de https://www.proquest.com/scholarly-journals/hacia-una-prospectivalatinoamericana-un-abordaje/docview/2326923105/se-2 accountid=37408 
Tegan Nuss, Belinda Morley, Maree Scully y Melanie Wakefield. (2021). El consumo de bebidas energéticas entre Adolescentes australianos asociados con un grupo de conductas alimentarias poco saludables y una duración corta del sueño. Nuss et al. Diario de nutrición. Obtenido de https://doi.org/10.1186/s12937-021-00719-Z

Thimphu, B. (17 al 20 de Febrero de 2016). "Construyendo sistemas alimentarios sostenibles y resilientes en Asia y el Pacífico. FAO FIAT PANIS, 1-14. doi:10.1007 / s12571-011-0149-9. 